\title{
The role of multidetector computed tomography for detecting aortic root pseudoanerysms complicating infective endocarditis
}

\author{
Edward TD Hoey', Sangeetha Rajoo² \\ Heart of England NHS Foundation Trust, Birmingham, UK \\ Correspondence: Edward TD Hoey. Address: Department of Radiology, Heart of England NHS Foundation Trust, \\ Birmingham, UK. Email: edwardhoey1@gmail.com
}

Received: January 15, 2014

Accepted: February 17, 2014

Online Published: February 25, 2014

DOI : 10.5430/ijdi.v1n1p13

URL: http://dx.doi.org/10.5430/ijdi.v1n1p13

\begin{abstract}
Echocardiography is the mainstay imaging technique for detecting infective endocarditis vegetations but may struggle to detect and characterise complicating features. There is increasing interest in the use of multidetector computed tomography (MDCT) as an adjunct to echocardiography, especially where there is concern for abscess or pseudoaneurysm formation. We present the case of a 70-year-old male with bio-prosthetic aortic valve endocarditis complicated by a large infected left ventricular outflow tract pseudoaneurysm which was diagnosed using MDCT angiography. Radiologists and clinicians should be aware of the emerging role of MDCT in this setting in order to advise and direct an appropriate imaging strategy.
\end{abstract}

\section{Keywords}

Computed tomography, Endocarditis, Pseudoaneurysm

\section{I ntroduction}

Infective endocarditis (IE) most commonly involves the aortic valve from where it can spread into the peri-annular soft tissues to cause abscesses, pseudoaneurysms and fistulas which can be difficult to define with echocardiography due to its limited soft tissue resolution. IE can also preferentially involve the left ventricular outflow tract (LVOT). Echocardiography is the mainstay imaging technique for detecting IE vegetations but may struggle to detect and characterise complicating features such as pseudoaneurysm formation. There is increasing interest in the use of multidetector computed tomography (MDCT) as an adjunct to echocardiography in some cases of IE, especially where there is concern for abscess or pseudoaneurysm formation. We present a case in which MDCT was central to the diagnosis of an infected pseudoanerysm arising from the LVOT and highlight its clinical utility for rapid diagnosis of endocarditis complications. Radiologists and clinicians should be aware of the emerging role of MDCT in this setting in order to advise and direct and appropriate imaging strategy in these patients. 


\section{Case report}

A 70-year-old man, with a history of bio-prosthetic aortic valve and root replacement 6-months previously (for aortic stenosis and aortic root aneurysm) presented to the emergency department with a 2-day history of a febrile illness. He described having rigors and feeling nauseous and lethargic. He was pyrexial at $39^{0}$ with a mildly elevated white cell count $\left(14 \times 10^{9} / l\right.$ (range 4-11)) and c-reactive protein level of $30 \mathrm{mg} / \mathrm{L}$ (range $0-5$ ). There was no murmur on auscultation and blood pressure was normal (130/65 mmHg). There were no signs of pulmonary oedema and no peripheral stigmata of infective endocarditis and but given the recent aortic surgery an urgent transesophageal echocardiogram (TEE) was performed. This showed a normal appearance of the aortic valve which was well seated with thin mobile leaflets and no vegetations. There was just trivial aortic regurgitation. There was however apparent expansion of the aorto-mitral continuity by a low echogenicity area with surrounding soft tissue thickening measuring $3.5 \mathrm{~cm} \times 1.5 \mathrm{~cm}$ which contained some areas of internal flow on Doppler interrogation. A clear point of communication with the cardiac chambers was not seen. In light of the TEE findings an urgent contrast medium enhanced multi-detector computed tomography (MDCT) study was performed to provide additional information. This showed a large abscess cavity within the aorto-mitral region which had a thin neck communicating through to the left ventricular outflow tract (LVOT), just inferior to the non-coronary aortic valve cusp (see Figure 1). There was no communication with the atria. Appearances were consistent with a large infected aortic root pseudoaneurysm. He was referred to cardiac surgery. Streptococcus sanguis was subsequently isolated from blood cultures.

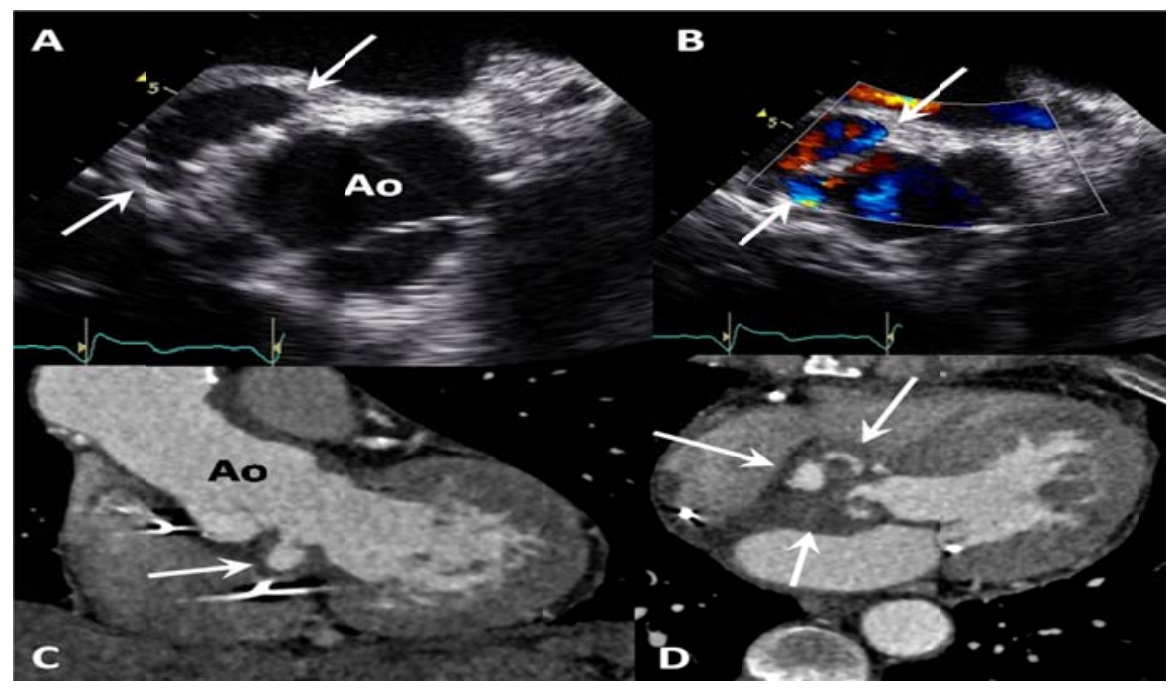

Figure 1. (A) Transesophageal echocardiogram image showing a collection adjacent to the aortic root (arrows). (B) Doppler interrogation of the collection shows some "swirling" (mixed red and blue coloured) internal flow consistent with a pseudoaneurysm (arrows). (C) Coronal oblique multidetector CT image showing a contrast medium filled out pouching in direct communication by a thin neck with the left ventricular outflow tract just below the line of the aortic valve annulus and inferior to the posterior aortic valve cusp (arrows). Appearances are typical for an infected psuedoaneurysm. (D) Axial multidetector CT image showing multiple pockets of contrast medium with surrounding soft tissue thickening and enhancement which is expanding the aorto-mitral continuity in keeping with a large complex infected pseudoaneurysm (arrows). Ao = aortic root.

\section{Discussion}

Infective endocarditis (IE) is a potentially life-threatening condition that frequently involves the aortic valve with streptococci and staphylococci account for $80 \%$ of cases $^{[1]}$. Major risk factors for endocarditis are congentital structural anomalies such as bicuspid aortic valve, prosthetic valves and intravenous drug use. Bacterial endocarditis causes leaflet 
destruction, perforation and incompetence. Aortic valve infection commonly spreads into the periannular soft tissues to cause abscesses, pseudoaneurysms and fistulas with the incidence of these complications reported to be $30 \%-50 \%{ }^{[2]}$. On rare occasions as in the presented case IE can localize its effects to the LVOT which is thought to be predisposed to by a longstanding aortic regurgitant jet causing damage of the endocardial surface ${ }^{[3]}$. IE of the LVOT is thought to have a particularly high likelihood of systemic embolisation which can lead to stroke and mycotic intracerebral abscess formation. Treatment of IE is usually with prolonged courses of intravenous antibiotics however surgical resection and valve replacement may be considered in the setting of severe aortic regurgitation, large valvular vegetations and perivalvular pseudoaneurysm/abscess or fistula ${ }^{[4]}$. The preoperative recognition of an infected pseudoaneurysm has important therapeutic implications requiring much more extensive surgical intervention with the need for debridement and often annulus reconstruction.

Diagnosis of IE is established using the revised Duke criteria which are a collection of major and minor criteria. Major criteria include a positive blood culture with typical IE microorganism, and evidence of endocardial involvement at echocardiography (e.g. oscillating valve or support structure vegetation or abscess) ${ }^{[5]}$. Minor criteria include a predisposing factor for IE (e.g. intravenous drug use), fever $>38^{\circ} \mathrm{C}$, evidence of systemic or pulmonary emboli (e.g splenic infarct) and immunological conditions such as glomerulonephritis ${ }^{[5]}$. The hallmark imaging sign of endocarditis are vegetations which are often small $(<5 \mathrm{~mm})$ and can be difficult to reliable detect with transthroacic echocardiography (TTE) which has a low sensitivity (detection rate of only $25 \%$ for small vegetations $)^{[1]}$. TEE has a reported sensitivity of $48 \%-100 \%$ for vegetations and is considered the reference imaging technique ${ }^{[1]}$. Perivalular pseudoaneurysms can however be difficult to define with TEE because of its rather limited soft tissue resolution and there is increasing interest in the use of MDCT.

Feuchtner et al. recently investigated MDCT for IE and its complications. They showed a high sensitivity (96\%) and specificity (97\%) for detection of leaflet vegetations $>4 \mathrm{~mm}$ and significantly improved accuracy of MDCT over TEE for detection and localization of perivalvular abscesses and pseudoaneyrms ${ }^{[2]}$. Gahide et al. reported a sensitivity and specificity of $100 \%$ and $87.5 \%$ for MDCT identification of aortic root pseudoaneurysms ${ }^{[3]}$.

The other main advantage of MDCT is the non-invasive assessment of coronary artery disease, as catheter coronary angiography, is not recommended in patients with IE due to the risk of inducing vegetation embolisation with catheter manipulation at invasive angiography ${ }^{[2]}$. On MDCT a vegetation appears as a low attenuation nodule, adherent to the valve surface. An abscess appears as irregularly shaped peripherally enhancing mass which is typically located within the periannular soft tissues but can also involve the myocardium or pericardial space. An infected pseudoaneurysm appears as a contrast medium filling outpouching that is in direct communication with the aortic root or left ventricular outflow tract $^{[2,4]}$.

Magnetic resonance imaging (MRI) is a widely used cardiac imaging technique which has established applications for assessment of structural heart disease. MRI however has a very limited role in the setting of IE due to its much inferior spatial resolution compared with MDCT $(1-2 \mathrm{~mm}$ vs $0.4 \mathrm{~mm})$ which makes small vegetations and abscesses very challenging to detect. The other main drawbacks of MRI are a prolonged examination time which in a critically ill patient may not be well tolerated and a contraindication to many types of patient monitoring and cardiovascular support equipment.

In conclusion MDCT is emerging as the imaging modality of choice for assessment of a suspected abscess or pseudoaneurysm complicating IE owing to its improved spatial resolution over that of TTE and TEE assessment. The clinical utility of MDCT for rapid diagnosis of perivalvular endocarditis complications should be appreciated in order to streamline the patient care pathway. 


\section{References}

[1] Mylonakis E, Calderwood SB. Infective endocarditis in adults. N Engl J Med. 2001 1; 345: 1318-30. http://dx.doi.org/10.1056/NEJMra010082

[2] Feuchtner GM, Stolzmann P, Dichtl W, et al. Multislice computed tomography in infective endocarditis: comparison with transesophageal echocardiography and intraoperative findings. J Am Coll Cardiol. 2009; 53: 436-44. http://dx.doi.org/10.1016/j.jacc.2008.01.077

[3] Iacovelli F, Scicchitano P, Zanna D, et al. Left ventricle outflow tract vegetation, embolism and troponin rise: an infective endocarditis case report. Intern Emerg Med. 2012 Sep; 7 Suppl 2: S145-7. http://dx.doi.org/10.1007/s11739-012-0761-5

[4] Gahide G, Bommart S, Demaria R, et al. Preoperative Evaluation in Aortic Endocarditis: Findings on Cardiac CT AJR Am J Roentgenol. 2010; 194: 574-8. http://dx.doi.org/10.2214/AJR.08.2120

[5] Li JS, Sexton DJ, Mick, N, et al. Proposed Modifications to the Duke Criteria for the Diagnosis of Infective Endocarditis. Clinical Infectious Diseases. 2000; 30: 633. http://dx.doi.org/10.1086/313753 